\title{
Conditioned avoidance decrement under spreading depression'
}

NELSON L, FREEDMAN, QUEEN'S UNIVERSITY, KINGSTON, ONTARIO LEONARD LASH, BUCKNELL UNIVERSITY

Bilateral applications of $\mathrm{KCl}$ to the exposed rat dura (SD) cause a profound loss of the ability to learn to perform a conditioned avoidance response (CAR). While under normal saline, latencies decreased rapidly over trials and within training sessions, but under SD those latencies actually increased. This debility under SD is attributed to some motor disability.

Bilateral spreading depression (SD) of the cortex typically yields a severe impairment of previously learned well trained conditioned avoidance responses (Bures et al, 1958; Tapp, 1962), and the impairment of learning a conditioned avoidance response (CAR) (Travis \& Sparks, 1963; Thompson, 1964,1965; Thompson \& Hjelle, 1965). This deficit in learning to perform a CAR has been attributed to a motor rather than a learning debility (Winocur, 1965).

The above experiments all report their findings in terms of trials to criterion or percent avoidance responses. The present problem is to find the transfer effects of SD training on performance with the cortex normal and vice versa using signal response latency as a measure. A second problem is to investigate the time course of SD effects within a training session when conditions are maximized for the learning of a CAR. Method

Forty male hooded rats (Long-Evans strain), between 3-4 mo. of age at the time of surgery, were used. For one week before surgery and during training, sodium sulfathiazole was available to each $S$ in his drinking water. The surgical procedure followed Tapp's (1962) chronic preparation. However, any $\mathrm{S}$ suffering dural insult or subdural hematoma at this time was not used in the behavioral phase of the experiment.

On the second post-operative day, $10 \mathrm{Ss}$ were assigned to each of four groups equated on the basis of pre-operative weight. The groups were differentiated by the treatments during the first two days of behavioral testing. On any one day Ss were run with either $\mathrm{KCl}$ or normal saline placed bilaterally on the dura. Some Ss received either $\mathrm{KCl}$ or saline on the first test day and either saline or $\mathrm{KCl}$ respectively on the second. On the third day of testing all groups received saline. This procedure not only allows between group comparisons but affords a study of transfer from zero, one and two days depression to behavior without depression; and from zero and one day behavior under saline to depressed behavior.

Spreading depression was induced and maintained during training by applying $24 \% \mathrm{KCl}$ bilaterally to S's dura
$15 \mathrm{~min}$. prior to the beginning of training and at $60-90$ min. intervals throughout the training period. Sterile normal saline was applied according to the same schedule to the duras of Ss whose cortex was not depressed on that day.

Conditioned avoidance training was conducted in an $11-1 / 2$ in. by $11-3 / 4$ in. long one-way shuttle box. A relay operated guillotine door separated the shock compartment from an equal sized goal compartment.

The relay was activated after $S$ was in the shock compartment and facing the door. Relay activation caused the door to drop (an auditory and visual stimulus) and started a loud standard 12-v buzzer (an auditory stimulus), and a response timer. Five sec. later a $1.3 \mathrm{ma}$ shock was delivered through the floor grids of the shock compartment. When $S$ entered the goal compartment a thyratron circuit terminated both the buzzer and the response timer. The guillotine door was then closed manually, $S$ was removed to a waiting cage and the next $S$ was placed in the shock compartment. The Ss were run one trial at a time in squads of four to six for 40 trials each day. The intertrial interval was thus maximally varied between about 4-6 min. depending on the squad size.

\section{Results and Discussion}

The number of days of training under $\operatorname{KCl}(0,1$, or 2$)$ preceding the first day of testing under saline did not significantly affect latency of responding on this day. All three groups had a significant $(p<.005)$ but undifferentiated decrease in latency over trials. Thus, there was no transfer from depressed to nondepressed responding.

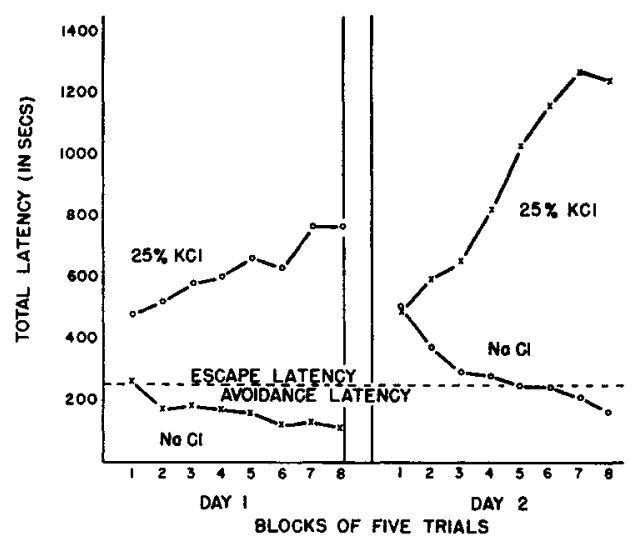

Fig. 1. Total latencies (10 $\mathrm{Ss}$ in each group) over blocks of five trials for Ss shifted from $25 \% \mathrm{KC1}$ on day 1 to normal saline (NaC1) on day 2 , and vice versa. 


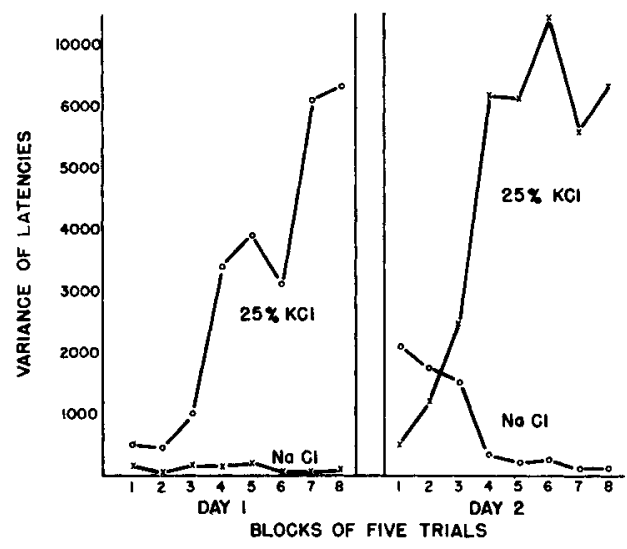

Fig. 2. Total variance of Ss' latencies over blocks of five trials for Ss shifted from $25 \% \mathrm{KC1}$ on day 1 to normal saline (NaC1) on day 2 , and vice versa.

Figure 1 shows total response latencies over blocks of five trials under $\mathrm{KCl}$ or saline. As expected, the latencies under saline are decreasing significantly $(p<.005)$ over trial blocks. Under $\mathrm{KCl}$ the latencies are not random, but increase significantly $(p<.005)$ over trial blocks. Again, no significant positive or negative transfer is evident for the group shifted from $\mathrm{KCl}$ on day 1 to saline on day 2 , or vice versa.

The variance of the Ss' latencies changes over trial blocks as well as the mean latencies. Figure 2 shows the variance increasing markedly over blocks in the $\mathrm{KCl}$ groups on both days. The increasing latencies and variances over trials in the $\mathrm{KCl}$ groups, but not the saline groups, are consistent with the notion of a general motor deficit reported from mean latencies by Travis \& Sparks (1963). Similar deficits were found in well trained avoidance responding by Tapp (1962) and Bures et al (1958).

On the other hand, Thompson has recently shown that Ss under SD can learn a CAR but more slowly than saline treated Ss. In a critical series of parametric studies Thompson (1965) and Thompson \& Hjelle (1965) demonstrated that much of the discrepancy in depression experiments was due to broad variability in procedures from one investigator to the next. Specifically, Thompson and Hjelle found that increasing CS discriminability and decreasing task complexity both decreased mean trials to criterion. However, Ss under SD were still inferior to saline treated Ss in all cases. Thompson has also shown that increasing the intertrial interval sec. significantly improves trials-to-criterion performance in bilaterally depressed animals, but again for all intervals the depressed animal is inferior to the saline control.

It should be clear that the optimizing parameters of CS discriminability, task complexity, and intertrial interval used by Thompson were also used in the present study. These studies differed mainly in the measures used to show avoidance conditioning, in the present case latency and in Thompson's case trials-to-criterion.

If the present results are attributable merely to failure to learn under spreading depression, it can be assumed that latencies in depressed animals over trials would be random and the variances would be homogeneous. Yet the present study finds neither random responding nor homogeneity, but significant latency increments and rising variance over trials. It is concluded therefore that some unspecified motor deficit is occurring in these animals.

\section{References}

Bures, J., Buresova, olga, \& Zaharova, A. Conditional reflexes and Leao's spreading cortical depression. J. comp. physiol. Psychol., 1958, 51, 263-268.

Tapp, J. T. Reversible cortical depression and avoidance behavior in the rat. J. comp. physiol. Psychol., 1962, 55, 306-308.

Thompson, R. W. Transfer of avoidance learning between normal and functionally elecorticate states. J. comp. physiol. Psychol., 1964, 57, 321-325.

Thompson, R. W. Effects of intertrial interval on avoidance learning in rats trained under bilateral spreading depressi n. Psychol. Rep., 1965, 16, 472-474.

Thompson, R. W., \& Hjelle, L. A. Effects of stimulus and response complexity on learning under bilateral spreading depression. $J$. comp. physiol. Psychol., 1965, 59, 122-124.

Travis, R. P., \& Sparks, D. L. The influence of unilateral and bilateral spreading depression during learning upon subsequent relearning. J. comp. physiol. Psychol., 1963, 56, 56-59.

Winocur, G. Bilateral spreading depression and avoidance learning in rats. Psychon. Sci., 1965, 3, 107-108.

\section{Note}

1. This research was supported by NSF Grant GB 1999 to Leonard Lash, NIMH Grant MH 02553 to R. S. Daniel, (University of Missouri), and PHS Predoctoral Fellowship to N. L. Freedman. The work was conducted at the University of Missouri. 